Article

\title{
Ipragliflozin Additively Ameliorates Non-Alcoholic Fatty Liver Disease in Patients with Type 2 Diabetes Controlled with Metformin and Pioglitazone: A 24-Week Randomized Controlled Trial
}

\author{
Eugene Han ${ }^{1}\left(\mathbb{D}\right.$, Yong-ho Lee ${ }^{2,3}$, Byung-Wan Lee ${ }^{2,3} \mathbb{C}^{\mathbb{D}}$, Eun Seok Kang ${ }^{2,3}$ and Bong-Soo Cha ${ }^{2,3, *}$ \\ 1 Division of Endocrinology, Department of Internal Medicine, Keimyung University School of Medicine, \\ Daegu 42601, Korea; jinsjins85@gmail.com \\ 2 Division of Endocrinology, Department of Internal Medicine, Yonsei University College of Medicine, \\ Seoul 03722, Korea; yholee@yuhs.ac (Y.-h.L.); bwanlee@yuhs.ac (B.-W.L.); edgo@yuhs.ac (E.S.K.) \\ 3 Institue of Endocrine Research, Yonsei University College of Medicine, Seoul 03722, Korea \\ * Correspondence: bscha@yuhs.ac; Tel.: +82-2-2228-1962; Fax: +82-2-393-6884
}

Received: 26 December 2019; Accepted: 15 January 2020; Published: 18 January 2020

\begin{abstract}
Despite the benefits of pioglitazone in the treatment of non-alcoholic fatty liver disease (NAFLD), many treated patients continue to experience disease progression. We aimed to investigate the additive effect of ipragliflozin on NAFLD in patients with type 2 diabetes treated with metformin and pioglitazone. In this 24-week randomized controlled trial, 44 patients with type 2 diabetes and comorbid NAFLD were either randomized to receive $50 \mathrm{mg}$ /day of ipragliflozin as an add-on treatment $(n=29)$ or maintained on metformin and pioglitazone $(n=15)$. The fatty burden was assessed using the fatty liver index, NAFLD liver fat score, and controlled attenuation parameter (CAP). Changes in fat and muscle depots were measured by dual-energy $\mathrm{x}$-ray absorptiometry and abdominal computed tomography scans. The enrolled patients were relatively controlled (mean baseline glycated hemoglobin of $6.6 \% \pm 0.6 \%$ ) and centrally obese (mean waist circumference of $101.6 \pm 10.9 \mathrm{~cm}$ ). At week 24, patients in the ipragliflozin add-on group exhibited reduced hepatic fat content (fatty liver index: $-9.8 \pm 1.9, p=0.002$; NAFLD liver fat score: $-0.5 \pm 0.2, p=0.049$; CAP: $\left.-8.2 \pm 7.8 \mathrm{~dB} / \mathrm{m}^{2}, p=0.133\right)$. Ipragliflozin add-on therapy also reduced whole-body visceral fat and the ratio of visceral to subcutaneous fat (change in whole-body visceral fat: $-69.6 \pm 21.5 \mathrm{~g}$; change in abdominal visceral fat: $-26.2 \pm 3.7 \mathrm{~cm}^{2}$; abdominal visceral to subcutaneous fat ratio: $-0.15 \pm 0.04$; all $p<0.05$ ). In conclusion, ipragliflozin treatment significantly ameliorates liver steatosis and reduces excessive fat in euglycemic patients with type 2 diabetes and NAFLD taking metformin and pioglitazone.
\end{abstract}

Keywords: non-alcoholic fatty liver disease; obesity; type 2 diabetes mellitus; sodium/glucose cotransporter 2 inhibitor

\section{Introduction}

As obesity and type 2 diabetes (T2D) emerge as comorbid endemic diseases, management strategies have shifted to account for obesity and T2D-related complications. Non-alcoholic fatty liver disease (NAFLD) is an obesity-related disease that is both a complication of and a risk factor for type 2 diabetes [1-3]. Traditionally, thiazolidinediones (TZDs) are the first-line treatment for patients with comorbid T2D and NAFLD [4]. TZDs are peroxisome proliferator-activated receptor gamma agonists that improve glucose tolerance and insulin sensitivity and enhance insulin action in the muscle, liver, and heart as well as in adipose tissue [5-7]. TZDs in patients with comorbid T2D and NAFLD 
have been shown to improve liver enzymes and hepatic histology [8]. Hepatic steatosis reduction independent of glycemic control has also been observed [5]. TZDs are ineffective in some patients with T2D and their use is associated with an increased risk for edema, fluid retention, and weight gain, particularly subcutaneous fat gain [9]. Moreover, the response rate to TZD treatment is relatively low (approximately $50 \%$ ) in NAFLD $[8,10,11]$.

Sodium/glucose cotransporter 2 (SGLT2) inhibitors have received significant attention due to their unique mechanism of action, whereby they inhibit glucose re-absorption independent of insulin [12]. Recent studies have reported the benefits of SGLT2 inhibitors in cardiovascular diseases $[13,14]$ and renal protection [15-17]. In addition, it has been demonstrated that SGLT2 inhibitors reduce visceral fat and attenuate inflammation [18]. Despite several studies in animal models and in patients with T2D [19], an understanding of how SGLT2 inhibitors affect NAFLD remains limited.

We hypothesized that ipragliflozin, an SGLT2 inhibitor, would reduce excessive fat in patients with comorbid T2D and NAFLD, and therefore serve as a candidate therapy for patients with NAFLD who are unresponsive to metformin and TZD combination therapy. The aim of this study, therefore, was to investigate changes in fat depots and the severity of NAFLD in patients taking ipragliflozin for 24 weeks in addition to metformin and pioglitazone compared to treatment with metformin and pioglitazone only.

\section{Methods}

\subsection{Study Design}

A randomized, 24-week, controlled, parallel, open-label study was conducted at the Severance Hospital, a tertiary university hospital in Seoul, Korea, from April 2016 to June 2017. We enrolled patients between 20 and 75 years of age with well-controlled type 2 diabetes (glycated hemoglobin (HbA1c) levels $\leq 9.5 \%$ or $80 \mathrm{mmol} / \mathrm{mol}$ ) and a body mass index (BMI) $\geq 23 \mathrm{~kg} / \mathrm{m}^{2}$ (cut-off for overweight according to the Asia-Pacific definition). Patients were eligible for the study if they were receiving metformin and pioglitazone combination therapy for at least 8 weeks and naive to treatment with any SGLT2 inhibitors. All patients exhibited NAFLD, as diagnosed by abdominal ultrasound and confirmed by radiologic specialists. The exclusion criteria for this study were: (1) diagnosis of type 1 diabetes, gestational diabetes, or any diabetes diagnosis other than type 2 diabetes; (2) history of addiction to alcohol, heavy alcohol consumption ( $\geq 210 \mathrm{~g} /$ week for men or $\geq 140 \mathrm{~g} /$ week for women), or patients with aspartate aminotransferase (AST), alanine aminotransferase (ALT), or bilirubin levels more than three times the upper normal limit; (3) other causes of liver disease (e.g., active viral or autoimmune hepatitis), liver cirrhosis, or hepatocellular carcinoma; (4) estimated glomerular filtration rate (eGFR) $<60 \mathrm{~mL} / \mathrm{min} / 1.73 \mathrm{~m}^{2}$; (5) medication associated with fatty liver disease (e.g., amiodarone, methotrexate, tamoxifen, or valproate) or weight loss; and (6) pregnant or nursing women. This study complied with the Declaration of Helsinki and Good Clinical Practice guidelines. The study protocol received ethical approval by the institutional review board at the Yonsei University College of Medicine (4-2015-1115). All subjects provided written informed consent. This study is described further at https://clinicaltrials.gov/ (NCT02875821).

Eligible patients were randomly assigned in a 1:2 ratio using a computer-generated randomization sequence to either receive $50 \mathrm{mg}$ /day of ipragliflozin as an add-on to metformin and pioglitazone combination treatment or were maintained on metformin and pioglitazone combination therapy. No dosage adjustments were made to any of the study medications in either study arm during the study period. All patients received diet and exercise counseling at the beginning of the study and were reminded at each study visit to follow their recommended plan.

\subsection{Laboratory and Imaging Studies}

All individuals underwent physical examination and clinical laboratory tests after an overnight $(\geq 8 \mathrm{~h}$ ) fasting period at baseline. Liver fat content was assessed via the controlled attenuation parameter 
(CAP). Briefly, the CAP measures ultrasonic attenuations at $3.5 \mathrm{MHz}$ using signals acquired by transient liver elastography (FibroScan ${ }^{\circledR} ;$ Echosens, Paris, France). The final CAP value was the median of each individual CAP value using the same valid measurements $[5,20]$. We also analyzed each patient's fatty liver index [21] and NAFLD liver fat score [22]. The equations are described in Table S1.

Whole-body fat distribution and muscle mass were measured via dual-energy $\mathrm{x}$-ray absorptiometry (DXA). An abdominal fat computed tomography (CT) scan (Tomoscan 350; Philips, Mahwah, NJ, USA) was performed to measure the abdominal subcutaneous fat area (SFA), abdominal visceral fat area (VFA), and ratio of VFA to SFA. Serial DXA examinations were performed in each patient using the same machine (QDR-4500W; Hologic, Bedford, MA, USA) throughout the study. The abdominal fat depot was measured by obtaining a single cross-sectional CT image of a 3-mm thick slice at the level of the L4-L5 interspace while the subject was in a supine position. The VFA and SFA were electronically calculated using the TeraRecon Aquarius software (Aquaris iNtuition Ver.4.4.6 TeraRecon, Foster City, CA, USA) over an attenuation range of 2150 to 250 Hounsfield units, as described previously [23,24]; the results herein are reported in $\mathrm{cm}^{2}$. The VFA was determined by measuring the intra-abdominal cavity at the internal aspect of the abdominal and oblique muscle walls surrounding the cavity and the posterior aspect of the vertebral body. The remaining fat interposed between the muscle and subcutaneous tissue was selected and calculated as the SFA. The ratio of VFA to SFA was calculated as VFA/(VFA + SFA).

Body weight, waist circumference, blood pressure, glycemic parameters (i.e., fasting plasma glucose (FPG) and $\mathrm{HbA1c}$ ), lipids (i.e., total cholesterol, high-density lipoprotein cholesterol (HDL-C), low-density lipoprotein cholesterol (LDL-C), and triglycerides), and liver enzymes (i.e., AST, ALT, and gamma glutamyl transferase $(\gamma-\mathrm{GT}))$ were measured at the start of the study and at weeks 12 and 24 .

\subsection{Outcomes}

The primary outcome was the change in total visceral fat as measured by DXA after 24 weeks. The key secondary outcomes were changes in CAP, fatty liver index, and NAFLD fatty liver score. In addition, we compared changes in SFA, VFA, SFA/VFA ratio, glycemic parameters, lipid profile, and liver enzymes at week 24. The homeostasis model assessment of insulin resistance (HOMA-IR) and HOMA- $\beta$ were quantified based on FPG and fasting insulin levels using the following calculations: HOMA-IR $=$ FPG $(\mathrm{mg} / \mathrm{dL}) \times$ insulin $(\mathrm{mIU} / \mathrm{L}) / 405$ and HOMA- $\beta=(360 \times$ insulin $(\mathrm{mIU} / \mathrm{L})) /(\mathrm{FPG}(\mathrm{mg} / \mathrm{dL})$ - 63)\%. The percent change $(\Delta)$ in each parameter was calculated as: (baseline value -24 -week value/baseline value $) \times 100(\%)$.

\subsection{Statistical Analysis}

The planned sample size was 45 subjects in a randomized 1:2 ratio $(n=15$ for metformin + pioglitazone and $n=30$ for metformin + pioglitazone + ipragliflozin), which was calculated a priori to have $90 \%$ power to detect a difference of $0.08 \mathrm{~kg}$ in visceral fat based on a standard deviation (SD) of $0.1 \mathrm{~kg}$ at $\alpha=0.05$ with a discontinuation rate of $10 \%$. The data are presented as the mean \pm SD for continuous variables and as the number or percent for categorical variables. We analyzed differences in participant characteristics between groups using paired t-tests for continuous variables and $\chi^{2}$ tests for categorical variables. The total cholesterol, triglyceride, HDL-C, LDL-C, AST, ALT, $\gamma$-GT, insulin, HOMA-IR, and HOMA- $\beta$ values were not normally distributed; analyses, therefore, were performed using log-transformed data. We tested treatment differences in the primary and key secondary endpoints using analysis of covariance (ANCOVA) models with treatment and sex as fixed effects and baseline values as covariates. To evaluate the association among changes in body weight, VFA, and muscle mass in the ipragliflozin add-on group, we performed Pearson's correlation analyses. A responder to ipragliflozin was defined as any individual who exhibited a decrease in body weight of more than $1.6 \mathrm{~kg}$ (median weight loss of the ipragliflozin group) after treatment. Multivariable-adjusted logistic regression analyses were performed to test the independent association between ipragliflozin 
response and other clinical factors. All statistical analyses were performed using IBM SPSS version 23.0 for Windows (IBM Corp., Armonk, NY, USA); $p<0.05$ was considered statistically significant.

\section{Results}

\subsection{Baseline Characteristics of the Study Population}

In total, 55 patients were screened and 45 patients with comorbid type 2 diabetes and NAFLD were enrolled. Glycemic parameters were stable (mean FPG $=119.6 \pm 20.9 \mathrm{mg} / \mathrm{L}$ and $\mathrm{HbA} 1 \mathrm{c}=6.6 \% \pm 0.6 \%$, $49.0 \pm 7.1 \mathrm{mmol} / \mathrm{mol}$ ), confirming the effectiveness of metformin + pioglitazone treatment in these patients (Table 1). Thirty subjects were randomly assigned to the ipragliflozin add-on group and 15 patients were assigned to the metformin + pioglitazone maintenance group. One patient withdrew consent during the study period; 44 patients completed the study through week 24 (Figure S1). The mean age of the patients was $53.9 \pm 10.9$ years, and $62.2 \%$ of the patients were male. The average time since diagnosis of type 2 diabetes was $9.4 \pm 5.8$ years, and $64.4 \%$ and $97.8 \%$ of patients had hypertension and dyslipidemia, respectively. The mean body weight was $83.3 \pm 14.6 \mathrm{~kg}$, and the mean BMI was $30.3 \pm 4.6 \mathrm{~kg} / \mathrm{m}^{2}$. Although all enrolled patients had NAFLD, their liver function test results were almost within normal limits $(37.6 \pm 39.4 \mathrm{IU} / \mathrm{L}, 27.7 \pm 15.4 \mathrm{IU} / \mathrm{L}$, and $32.3 \pm 21.5 \mathrm{IU} / \mathrm{L}$ for $\gamma$-GT, AST, and ALT, respectively). The mean CAP was $306.3 \pm 38.3 \mathrm{~dB} / \mathrm{m}$. The mean fatty liver index score was $29.9 \pm 20.3$ and the mean NAFLD liver fat score was $-1.9 \pm 1.4$. The mean VFA-to-SFA ratio was $47.0 \pm 11.8 \%$. The mean values for total fat mass, total fat ratio, estimated visceral fat, and total muscle mass were $25.3 \pm 7.8 \mathrm{~kg}, 29.8 \% \pm 6.9 \%, 683.5 \pm 173.4 \mathrm{~g}$, and $56.3 \pm 11.2 \mathrm{~kg}$, respectively. Baseline characteristics were similar between the two groups.

Table 1. Baseline characteristics of the study population.

\begin{tabular}{|c|c|c|c|}
\hline Parameters & $\begin{array}{c}\text { Metformin }+ \\
\text { Pioglitazone }(n=15)\end{array}$ & $\begin{array}{c}\text { Metformin }+ \\
\text { Pioglitazone }+ \\
\text { Ipragliflozin }(n=30)\end{array}$ & $p$-Value \\
\hline Age (years) & $56.7 \pm 11.8$ & $52.5 \pm 10.3$ & 0.233 \\
\hline Sex (male), $N(\%)$ & $9(60.0)$ & $19(63.3)$ & $>0.999$ \\
\hline Diabetes duration (years) & $10.1 \pm 5.6$ & $9.1 \pm 6.0$ & 0.563 \\
\hline Waist circumference $(\mathrm{cm})$ & $100.5 \pm 7.7$ & $102.1 \pm 12.2$ & 0.606 \\
\hline Weight (kg) & $81.4 \pm 8.5$ & $84.2 \pm 16.9$ & 0.471 \\
\hline $\operatorname{BMI}\left(\mathrm{kg} / \mathrm{m}^{2}\right)$ & $30.2 \pm 2.5$ & $30.4 \pm 5.4$ & 0.871 \\
\hline $\mathrm{SBP}(\mathrm{mmHg})$ & $124.9 \pm 9.2$ & $125.3 \pm 11.1$ & 0.889 \\
\hline $\mathrm{DBP}(\mathrm{mmHg})$ & $74.0 \pm 9.0$ & $75.6 \pm 10.0$ & 0.605 \\
\hline HTN, N (\%) & $11(73.3)$ & $18(60.0)$ & 0.378 \\
\hline Dyslipidemia, $N(\%)$ & $15(100.0)$ & $29(96.7)$ & $>0.999$ \\
\hline FPG $(\mathrm{mg} / \mathrm{dL})$ & $118.4 \pm 19.7$ & $120.1 \pm 21.8$ & 0.790 \\
\hline $\mathrm{HbA} 1 \mathrm{c}(\%)$ & $6.6 \pm 0.6$ & $6.7 \pm 0.7$ & 0.592 \\
\hline $\mathrm{HbA} 1 \mathrm{c}(\mathrm{mmol} / \mathrm{mol})$ & $48.2 \pm 6.4$ & $49.4 \pm 7.5$ & 0.598 \\
\hline HOMA-IR * & $3.4 \pm 2.8$ & $2.7 \pm 1.8$ & 0.351 \\
\hline HOMA- $\beta(\%) *$ & $73.5 \pm 38.0$ & $69.0 \pm 59.4$ & 0.382 \\
\hline Total cholesterol (mg/dL) & $170.3 \pm 21.7$ & $186.7 \pm 34.9$ & 0.110 \\
\hline HDL cholesterol (mg/dL) & $49.9 \pm 11.9$ & $50.2 \pm 13.3$ & 0.960 \\
\hline Triglycerides (mg/dL) & $152.5 \pm 91.8$ & $161.3 \pm 66.3$ & 0.551 \\
\hline LDL cholesterol (mg/dL) & $89.8 \pm 17.3$ & $104.2 \pm 28.8$ & 0.103 \\
\hline $\mathrm{WBC}\left(10^{3} / \mu \mathrm{L}\right)$ & $6.9 \pm 1.1$ & $7.1 \pm 2.0$ & 0.661 \\
\hline Hemoglobin (mg/dL) & $13.7 \pm 1.5$ & $14.1 \pm 1.4$ & 0.408 \\
\hline Platelet $\left(10^{9} / \mathrm{L}\right) *$ & $239.5 \pm 52.4$ & $257.3 \pm 59.1$ & 0.398 \\
\hline Creatinine $(\mathrm{mg} / \mathrm{dL})$ & $0.8 \pm 0.2$ & $0.7 \pm 0.2$ & 0.381 \\
\hline eGFR, EPI (mL/min/1.73 m²) & $94.8 \pm 10.1$ & $101.9 \pm 11.8$ & 0.053 \\
\hline Uric acid $(\mathrm{mg} / \mathrm{dL})$ & $5.3 \pm 1.3$ & $5.5 \pm 1.5$ & 0.692 \\
\hline Albumin (g/dL) & $4.3 \pm 0.3$ & $4.4 \pm 0.2$ & 0.633 \\
\hline
\end{tabular}


Table 1. Cont.

\begin{tabular}{|c|c|c|c|}
\hline Parameters & $\begin{array}{c}\text { Metformin }+ \\
\text { Pioglitazone }(n=15)\end{array}$ & $\begin{array}{c}\text { Metformin }+ \\
\text { Pioglitazone }+ \\
\text { Ipragliflozin }(n=30)\end{array}$ & $p$-Value \\
\hline$\gamma$-GT $(\mathrm{IU} / \mathrm{L}) *$ & $31.8 \pm 15.6$ & $40.5 \pm 47.0$ & 0.677 \\
\hline $\operatorname{AST}(\mathrm{IU} / \mathrm{L})$ * & $30.4 \pm 19.6$ & $26.3 \pm 12.9$ & 0.433 \\
\hline $\operatorname{ALT}(\mathrm{IU} / \mathrm{L}) *$ & $31.1 \pm 13.5$ & $32.9 \pm 24.8$ & 0.779 \\
\hline Transient elastography, CAP $(\mathrm{dB} / \mathrm{m})$ & $307.7 \pm 37.0$ & $305.5 \pm 39.5$ & 0.858 \\
\hline \multicolumn{4}{|l|}{ NAFLD score } \\
\hline Fatty liver index & $66.5 \pm 18.7$ & $68.2 \pm 21.3$ & 0.786 \\
\hline NAFLD liver fat score & $0.9 \pm 1.4$ & $0.6 \pm 1.5$ & 0.518 \\
\hline \multicolumn{4}{|l|}{ Abdominal fat CT scan } \\
\hline VFA $\left(\mathrm{cm}^{2}\right)$ & $223.3 \pm 90.8$ & $203.0 \pm 55.0$ & 0.528 \\
\hline $\mathrm{SFA}\left(\mathrm{cm}^{2}\right)$ & $230.1 \pm 80.6$ & $213.3 \pm 111.0$ & 0.336 \\
\hline Ratio of VFA to SFA (\%) & $49.0 \pm 11.4$ & $46.1 \pm 12.2$ & 0.422 \\
\hline \multicolumn{4}{|l|}{ DXA scan } \\
\hline Total fat mass $(\mathrm{kg})$ & $25.0 \pm 7.7$ & $24.7 \pm 7.0$ & 0.869 \\
\hline Total fat ratio $(\%)$ & $29.6 \pm 7.7$ & $29.6 \pm 6.5$ & 0.857 \\
\hline Estimated visceral fat (g) & $664.1 \pm 190.5$ & $690.8 \pm 169.1$ & 0.600 \\
\hline Total muscle mass (kg) & $55.8 \pm 9.9$ & $56.6 \pm 12.1$ & 0.832 \\
\hline
\end{tabular}

* Log-transformed. Data for continuous variables were expressed as mean \pm SD for parametric variables. Abbreviations: ALT, alanine transaminase; AST, aspartate aminotransferase; BMI, body mass index; CAP, controlled attenuation parameter; CT, computed tomography; DBP, diastolic blood pressure; DXA, dual-energy x-ray absorptiometry; eGFR, estimated glomerular filtration rate; EPI, epidemiology collaboration equation; FPG, fasting plasma glucose; HbA1c, glycated hemoglobin; HDL, high-density lipoprotein; HTN, hypertension; $\gamma$-GT, gamma glutamyl transferase; HOMA-IR, homeostatic model assessment of insulin resistance; LDL, low-density lipoprotein; NAFLD, non-alcoholic fatty liver disease; SBP, systolic blood pressure; SFA, subcutaneous fat area; VFA, visceral fat area; WBC, white blood cell.

\subsection{Effect of Ipragliflozin Add-On Treatment on Body Weight and Fat Depots}

Body weight, BMI, and waist circumference were significantly decreased in the ipragliflozin add-on group at 24 weeks compared to baseline $\left(-1.6 \pm 0.4 \mathrm{~kg},-0.6 \pm 0.1 \mathrm{~kg} / \mathrm{m}^{2}\right.$, and $-3.2 \pm 0.8 \mathrm{~cm}$, respectively; all $p<0.05$ ) (Table 2). Reductions in body weight and BMI were initially observed at 12 weeks and continued to decrease through 24 weeks (Figure 1A,B). The waist circumference was reduced in the ipragliflozin add-on group at week 24 only (adjusted mean difference of $-2.71 \%, 95 \%$ $\mathrm{CI}=-4.65$ to $-0.78, p=0.001$ ) (Figure $1 \mathrm{C}$ ).

Table 2. Changes in glycemic, hepatic, and lipid parameters compared between baseline and week 24 by treatment group.

\begin{tabular}{cccc}
\hline & $\begin{array}{c}\text { Metformin }+ \\
\text { Pioglitazone }(\boldsymbol{n}=\mathbf{1 5})\end{array}$ & $\begin{array}{c}\text { Metformin }+ \\
\text { Pioglitazone }+ \\
\text { Ipragliflozin }(\boldsymbol{n}=\mathbf{2 9})\end{array}$ & $p$-Value \\
\hline Total VAT (g) & & & \\
Baseline & $664.1 \pm 190.5$ & $698.0 \pm 167.6$ & 0.547 \\
Week 24 & $686.4 \pm 185.3$ & $626.4 \pm 198.9$ & 0.338 \\
Changes from baseline & $22.3 \pm 40.1$ & $\mathbf{- 7 1 . 5} \pm \mathbf{2 1 . 5}$ & $\mathbf{0 . 0 2 9}$ \\
\hline Total fat (kg) & & & \\
Baseline & $25.0 \pm 7.6$ & $25.8 \pm 8.0$ & 0.766 \\
Week 24 & $24.3 \pm 5.6$ & $\mathbf{2 4 . 7 \pm 8 . 2}$ & 0.849 \\
Changes from baseline & $0.7 \pm 1.3$ & $\mathbf{- 1 . 0 \pm 0 . 3}$ & 0.774 \\
\hline Total muscle (kg) & & & \\
Baseline & $55.8 \pm 9.9$ & $56.6 \pm 12.1$ & 0.832 \\
Week 24 & $56.1 \pm 1.0$ & $55.9 \pm 11.4$ & 0.970 \\
Changes from baseline & $0.2 \pm 0.3$ & $\mathbf{- 0 . 8}$ & 0.079 \\
\hline
\end{tabular}


Table 2. Cont.

\begin{tabular}{|c|c|c|c|}
\hline & $\begin{array}{c}\text { Metformin }+ \\
\text { Pioglitazone }(n=15)\end{array}$ & $\begin{array}{c}\text { Metformin }+ \\
\text { Pioglitazone }+ \\
\text { Ipragliflozin }(n=29)\end{array}$ & $p$-Value \\
\hline \multicolumn{4}{|l|}{ VFA $\left(\mathrm{cm}^{2}\right)$} \\
\hline Baseline & $223.3 \pm 90.8$ & $209.1 \pm 63.3$ & 0.546 \\
\hline Week 24 & $230.3 \pm 87.6$ & $182.9 \pm 63.7$ & 0.046 \\
\hline Changes from baseline & $7.0 \pm 7.7$ & $-26.2 \pm 3.7 * *$ & $<0.001$ \\
\hline \multicolumn{4}{|l|}{ SFA $\left(\mathrm{cm}^{2}\right)$} \\
\hline Baseline & $230.1 \pm 80.6$ & $267.5 \pm 115.4$ & 0.269 \\
\hline Week 24 & $228.7 \pm 90.0$ & $258.2 \pm 99.7$ & 0.341 \\
\hline Changes from baseline & $-1.4 \pm 5.0$ & $-9.3 \pm 7.2$ & 0.460 \\
\hline \multicolumn{4}{|l|}{$\mathrm{CAP}(\mathrm{dB} / \mathrm{m})$} \\
\hline Baseline & $307.7 \pm 37.0$ & $306.6 \pm 39.8$ & 0.928 \\
\hline Week 24 & $319.5 \pm 44.8$ & $298.6 \pm 45.2$ & 0.207 \\
\hline Changes from baseline & $11.7 \pm 12.1$ & $-8.0 \pm 8.5$ & 0.182 \\
\hline \multicolumn{4}{|l|}{ Body weight (kg) } \\
\hline Baseline & $81.4 \pm 8.5$ & $84.3 \pm 17.2$ & 0.470 \\
\hline Week 12 & $81.8 \pm 8.4$ & $82.8 \pm 17.1$ & 0.780 \\
\hline Week 24 & $81.9 \pm 7.6$ & $82.6 \pm 16.9$ & 0.854 \\
\hline Changes from baseline & $0.4 \pm 0.6$ & $-1.6 \pm 0.4^{* *}$ & 0.003 \\
\hline \multicolumn{4}{|l|}{ BMI $\left(\mathrm{kg} / \mathrm{m}^{2}\right)$} \\
\hline Baseline & $30.2 \pm 2.5$ & $30.6 \pm 5.3$ & 0.734 \\
\hline Week 12 & $30.3 \pm 2.5$ & $30.1 \pm 5.4$ & 0.850 \\
\hline Week 24 & $30.4 \pm 2.6$ & $30.1 \pm 5.3$ & 0.745 \\
\hline Changes from baseline & $0.2 \pm 0.2$ & $-0.6 \pm 0.1 * *$ & 0.001 \\
\hline \multicolumn{4}{|l|}{$\begin{array}{l}\text { Waist circumference } \\
(\mathrm{cm})\end{array}$} \\
\hline Baseline & $100.5 \pm 7.7$ & $102.4 \pm 12.3$ & 0.542 \\
\hline Week 12 & $99.9 \pm 8.4$ & $101.2 \pm 11.4$ & 0.698 \\
\hline Week 24 & $100.0 \pm 8.2$ & $99.2 \pm 11.6$ & 0.815 \\
\hline Changes from baseline & $0.5 \pm 0.7$ & $-3.2 \pm 0.8^{*}$ & 0.038 \\
\hline \multicolumn{4}{|l|}{ SBP (mmHg) } \\
\hline Baseline & $124.9 \pm 9.2$ & $125.8 \pm 11.1$ & 0.790 \\
\hline Week 12 & $121.7 \pm 9.6$ & $124.6 \pm 9.2$ & 0.337 \\
\hline Week 24 & $128.1 \pm 9.6$ & $125.1 \pm 10.8$ & 0.382 \\
\hline Changes from baseline & $3.2 \pm 2.0$ & $-0.6 \pm 2.1$ & 0.242 \\
\hline \multicolumn{4}{|l|}{ DBP (mmHg) } \\
\hline Baseline & $74.0 \pm 9.0$ & $75.5 \pm 10.2$ & 0.622 \\
\hline Week 12 & $72.1 \pm 7.8$ & $77.8 \pm 9.9$ & 0.062 \\
\hline Week 24 & $75.0 \pm 8.5$ & $77.7 \pm 8.5$ & 0.449 \\
\hline Changes from baseline & $1.6 \pm 2.5$ & $2.1 \pm 1.6$ & 0.868 \\
\hline \multicolumn{4}{|l|}{ FPG (mg/dL) } \\
\hline Baseline & $118.4 \pm 19.7$ & $121.2 \pm 21.3$ & 0.674 \\
\hline Week 12 & $140.7 \pm 39.2 *$ & $125.5 \pm 20.6$ & 0.180 \\
\hline Week 24 & $116.3 \pm 20.9$ & $117.5 \pm 19.8$ & 0.846 \\
\hline Changes from baseline & $-2.1 \pm 8.0$ & $-3.7 \pm 3.4$ & 0.835 \\
\hline \multicolumn{4}{|l|}{ HbA1c (\%) } \\
\hline Baseline & $6.6 \pm 0.6$ & $6.7 \pm 0.7$ & 0.595 \\
\hline Week 12 & $6.9 \pm 0.9$ & $6.4 \pm 0.5$ & 0.035 \\
\hline Week 24 & $6.8 \pm 0.7^{*}$ & $6.5 \pm 0.7$ & 0.287 \\
\hline Changes from baseline & $0.2 \pm 0.1$ & $-0.1 \pm 0.2 *$ & 0.129 \\
\hline
\end{tabular}


Table 2. Cont.

\begin{tabular}{|c|c|c|c|}
\hline & $\begin{array}{c}\text { Metformin }+ \\
\text { Pioglitazone }(n=15)\end{array}$ & $\begin{array}{c}\text { Metformin }+ \\
\text { Pioglitazone }+ \\
\text { Ipragliflozin }(n=29)\end{array}$ & $p$-Value \\
\hline \multicolumn{4}{|l|}{ HOMA-IR } \\
\hline Baseline & $3.4 \pm 2.8$ & $2.7 \pm 1.8$ & 0.273 \\
\hline Week 24 & $3.5 \pm 2.5$ & $2.2 \pm 1.4$ & 0.050 \\
\hline Changes from baseline & $0.1 \pm 0.8$ & $-0.5 \pm 0.2 *$ & 0.400 \\
\hline \multicolumn{4}{|l|}{ HOMA- $\beta$} \\
\hline Baseline & $73.5 \pm 38.0$ & $66.6 \pm 59.0$ & 0.182 \\
\hline Week 24 & $112.7 \pm 151.1$ & $55.7 \pm 41.2$ & 0.039 \\
\hline Changes from baseline & $39.1 \pm 37.4$ & $-10.9 \pm 8.4$ & 0.212 \\
\hline \multicolumn{4}{|l|}{ AST (IU/L) } \\
\hline Baseline & $30.4 \pm 19.6$ & $26.6 \pm 13.0$ & 0.485 \\
\hline Week 12 & $28.5 \pm 14.4$ & $27.8 \pm 16.8$ & 0.781 \\
\hline Week 24 & $24.7 \pm 10.0$ & $24.3 \pm 10.6$ & 0.845 \\
\hline Changes from baseline & $5.7 \pm 3.2$ & $2.3 \pm 1.1$ & 0.323 \\
\hline \multicolumn{4}{|l|}{ ALT (IU/L) } \\
\hline Baseline & $31.1 \pm 13.5$ & $33.4 \pm 25.1$ & 0.839 \\
\hline Week 12 & $27.3 \pm 8.8$ & $31.0 \pm 20.9$ & 0.781 \\
\hline Week 24 & $26.5 \pm 11.8$ & $25.6 \pm 16.9$ & 0.511 \\
\hline Changes from baseline & $-4.7 \pm 2.9$ & $-7.8 \pm 2.6^{* *}$ & 0.458 \\
\hline \multicolumn{4}{|l|}{$\gamma$-GT (IU/L) } \\
\hline Baseline & $31.8 \pm 15.6$ & $40.9 \pm 47.8$ & 0.671 \\
\hline Week 24 & $29.9 \pm 13.8$ & $29.7 \pm 24.6$ & 0.510 \\
\hline Changes from baseline & $-1.9 \pm 2.0$ & $-11.2 \pm 4.9 * *$ & 0.189 \\
\hline \multicolumn{4}{|l|}{$\begin{array}{c}\text { Total cholesterol } \\
(\mathrm{mg} / \mathrm{dL})\end{array}$} \\
\hline Baseline & $170.3 \pm 21.7$ & $187.1 \pm 35.4$ & 0.109 \\
\hline Week 12 & $170.9 \pm 30.1$ & $183.4 \pm 33.2$ & 0.260 \\
\hline Week 24 & $158.4 \pm 23.2$ & $184.6 \pm 35.6$ & 0.012 \\
\hline Changes from baseline & $-11.9 \pm 5.8$ & $-2.5 \pm 3.6$ & 0.158 \\
\hline \multicolumn{4}{|l|}{ HDL (mg/dL) } \\
\hline Baseline & $50.0 \pm 11.9$ & $50.7 \pm 13.2$ & 0.853 \\
\hline Week 12 & $51.0 \pm 16.2$ & $51.1 \pm 12.2$ & 0.742 \\
\hline Week 24 & $49.2 \pm 13.0$ & $52.4 \pm 10.6$ & 0.271 \\
\hline Changes from baseline & $-0.7 \pm 1.0$ & $1.7 \pm 1.6$ & 0.302 \\
\hline \multicolumn{4}{|l|}{ Triglyceride (mg/dL) } \\
\hline Baseline & $152.5 \pm 91.8$ & $159.7 \pm 66.9$ & 0.612 \\
\hline Week 12 & $177.0 \pm 135.5$ & $166.5 \pm 99.1$ & 0.825 \\
\hline Week 24 & $163.5 \pm 106.2$ & $149.0 \pm 56.4$ & 0.744 \\
\hline Changes from baseline & $11.0 \pm 13.9$ & $-10.8 \pm 11.6$ & 0.258 \\
\hline \multicolumn{4}{|l|}{ LDL (mg/dL) } \\
\hline Baseline & $91.3 \pm 16.9$ & $102.8 \pm 28.9$ & 0.106 \\
\hline Week 12 & $92.0 \pm 19.4$ & $100.8 \pm 29.1$ & 0.469 \\
\hline Week 24 & $82.0 \pm 20.8$ & $102.4 \pm 29.4$ & 0.024 \\
\hline Changes from baseline & $-9.3 \pm 3.7 * *$ & $2.1 \pm 2.7$ & 0.130 \\
\hline
\end{tabular}

Data are mean $\pm \mathrm{SD} ;{ }^{*} p \leq 0.05$ compared with baseline, ${ }^{* *} p \leq 0.05$ compared with baseline. Abbreviations: $\mathrm{ALT}$, alanine transaminase; AST, aspartate aminotransferase; BMI, body mass index; CAP, controlled attenuation parameter; DBP, diastolic blood pressure; DXA, dual-energy x-ray absorptiometry; FLI, fatty liver index; FPG, fasting plasma glucose; HbA1c, glycated hemoglobin; HDL, high-density lipoprotein; $\gamma$-GT, gamma glutamyl transferase; HOMA-IR, homeostatic model assessment of insulin resistance; LDL, low-density lipoprotein; LFS, non-alcoholic fatty liver fat score; NAFLD, non-alcoholic fatty liver disease; SBP, systolic blood pressure; SFA, subcutaneous fat area; VFA, visceral fat area. 

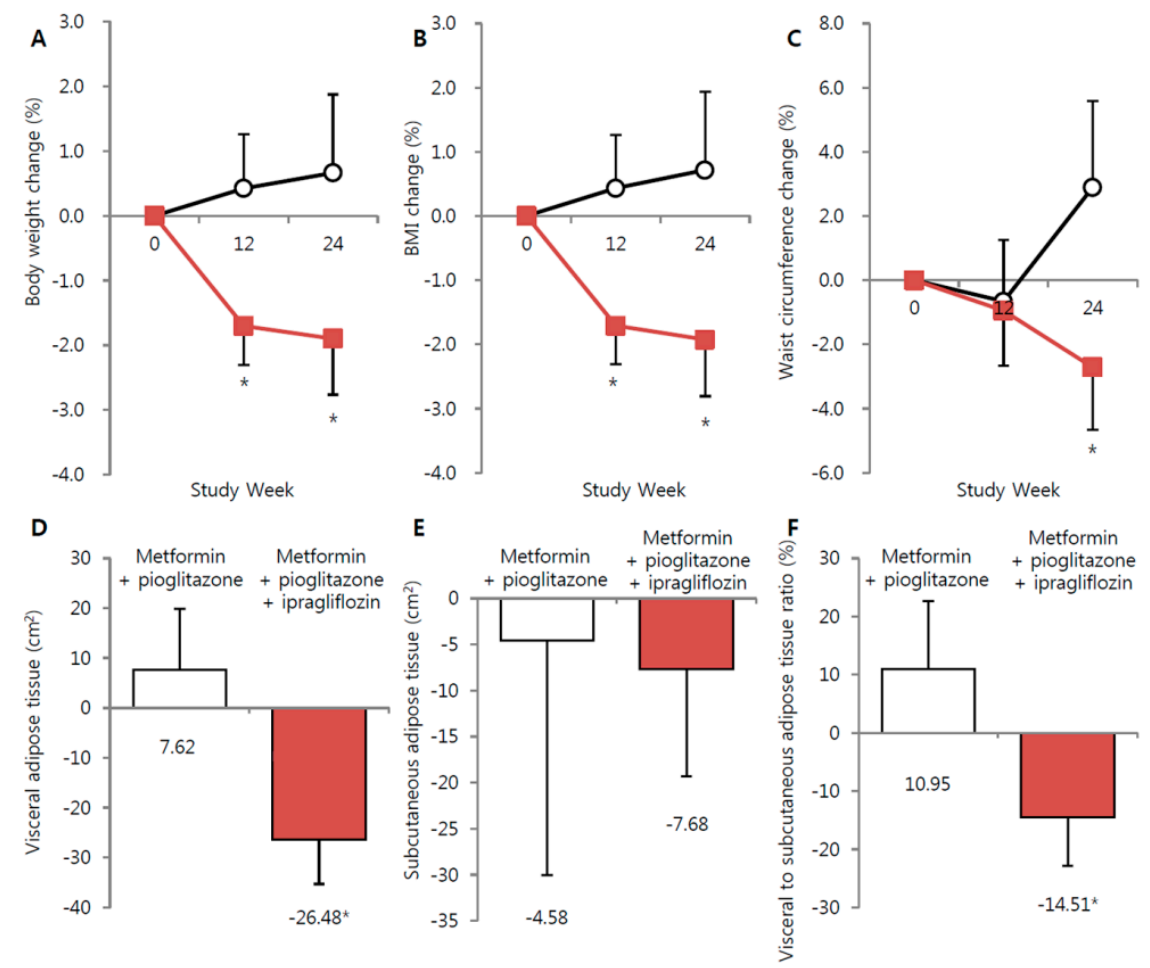

Figure 1. Changes in body weight, body mass index, waist circumference, abdominal adipose tissue, body fat and muscle from baseline to week 24. (A) Body weight. (B) Body mass index. (C) Waist circumference. (D) Abdominal visceral adipose tissue assessed by CT. (E) Abdominal subcutaneous adipose tissue assessed by CT. (F) Abdominal visceral to subcutaneous adipose tissue ratio assessed by CT. Red square: metformin + pioglitazone + ipragliflozin. Black circle: metformin + pioglitazone. * $p<0.05$.

A statistically significant reduction in total visceral fat was observed in the ipragliflozin add-on group at 24 weeks compared to the metformin + pioglitazone maintenance group (mean change in total visceral fat: $-69.64 \mathrm{~g}(95 \% \mathrm{CI}=-117.59$ to $-20.78 \mathrm{~g})$ versus $17.83 \mathrm{~g}(95 \% \mathrm{CI}=-49.58$ to $85.23 \mathrm{~g})$, ipragliflozin add-on versus metformin + pioglitazone maintenance groups, respectively). The VFA and VFA-to-SFA ratio were also decreased in the ipragliflozin add-on group (adjusted change in mean VFA: $-26.57 \mathrm{~cm}^{2}\left(95 \% \mathrm{CI}=-35.35\right.$ to $\left.-17.79 \mathrm{~cm}^{2}\right)$ versus $7.79 \mathrm{~cm}^{2}\left(95 \% \mathrm{CI}=-4.44\right.$ to $\left.20.02 \mathrm{~cm}^{2}\right)$; adjusted change in mean VFA-to-SFA ratio: $-0.15 \%(95 \% \mathrm{CI}=-0.23 \%$ to $-0.07 \%)$ versus $0.12 \%(95 \% \mathrm{CI}=0.00 \%$ to $0.23 \%$ ); ipragliflozin add-on versus metformin + pioglitazone maintenance group, respectively) (Figure 1D-F). SFA decreased in both groups, and total muscle mass decreased in the ipragliflozin group, but these changes were not statistically significant.

\subsection{Effect of Ipragliflozin Treatment on NAFLD, Glycemic, and Lipid Parameters}

The NAFLD surrogate marker, ALT, was significantly decreased in the ipragliflozin group (from $33.4 \pm 25.1 \mathrm{IU} / \mathrm{L}$ to $25.6 \pm 16.9 \mathrm{IU} / \mathrm{L}$ and from $31.1 \pm 13.5 \mathrm{IU} / \mathrm{L}$ to $26.5 \pm 11.8 \mathrm{IU} / \mathrm{L}$ in the ipragliflozin add-on and metformin + pioglitazone maintenance groups, respectively; $p<0.001$ ) Similarly, $\gamma$-GT was significantly decreased in the ipragliflozin group (from $40.9 \pm 47.8 \mathrm{IU} / \mathrm{L}$ to $29.7 \pm 24.6 \mathrm{IU} / \mathrm{L}$ and from $31.8 \pm 15.6 \mathrm{IU} / \mathrm{L}$ to $29.9 \pm 13.8 \mathrm{IU} / \mathrm{L}$ in the ipragliflozin add-on and metformin + pioglitazone maintenance groups, respectively; $p<0.001$ ) (Table 2). Both the fatty liver index and the NAFLD liver fat score were significantly reduced in the ipragliflozin add-on group $(-9.80,95 \% \mathrm{CI}=-13.7$ to -6.0 ; and $-0.54,95 \% \mathrm{CI}=-0.86$ to -0.23 , respectively), but not in the metformin + pioglitazone maintenance group $(1.05,95 \% \mathrm{CI}=-4.32$ to 6.42 ; and $0.01,95 \%=-0.44$ to 0.44 , respectively) (Figure 2 ). The adjusted mean change in CAP exhibited a non-significant decline in only the ipragliflozin add-on group ( $-7.98 \mathrm{~dB} / \mathrm{m} ; 95 \% \mathrm{CI}=-9.96$ to 33.2$)$. FPG and HbA1c levels also were slightly decreased in the 
ipragliflozin add-on group, but no significant difference between the two groups was observed. In addition, HOMA-IR improved in the ipragliflozin add-on group (from $2.7 \pm 1.8$ to $2.2 \pm 1.4$ ). Patients in the metformin + pioglitazone maintenance group exhibited a greater reduction in total cholesterol and LDL-C, whereas patients in the ipragliflozin add-on group exhibited a non-significant elevation in both HDL-C and LDL-C despite a reduction in total cholesterol.
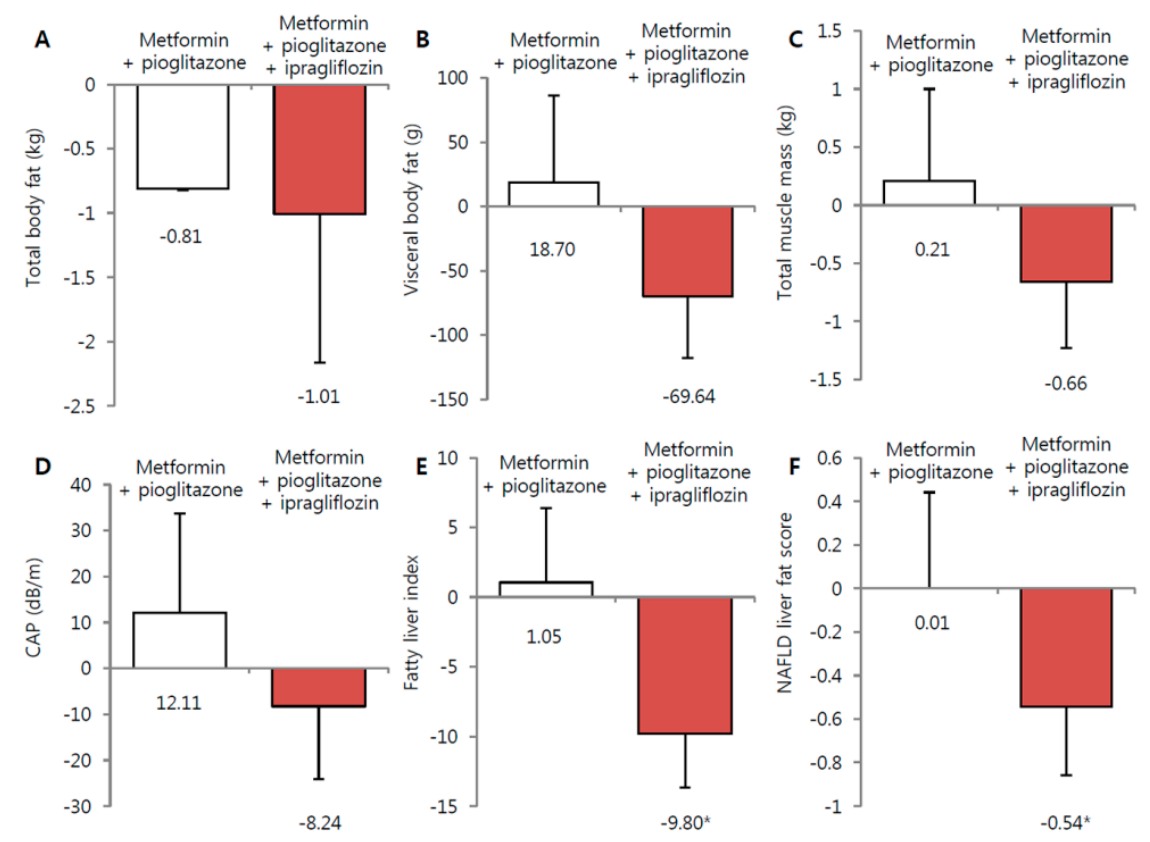

Figure 2. Changes in body fat, muscle and hepatic fat contents from baseline to week 24. (A) Total body fat. (B) Visceral body fat. (C) Total muscle mass by dual-energy x-ray absorptiometry. (D) Controlled attenuation parameter assessed by transient liver elastography. (E) Fatty liver index. (F) Non-alcoholic fatty liver disease liver fat score. Red square: metformin + pioglitazone + ipragliflozin. Black circle: metformin + pioglitazone. ${ }^{*} p<0.05$.

\subsection{Correlation between Changes in Body Weight, VFA, and Muscle Mass Following Ipragliflozin Add-On Treatment}

Body weight, visceral fat, and muscle mass were reduced in the ipragliflozin add-on group. We, therefore, explored the associations between changes in body weight, VFA, and muscle mass (Figure 3). The reduction in body weight had a significant moderate positive correlation with the change in VFA (Pearson's correlation coefficient $=0.422, p=0.023$ ) and with the change in CAP (Pearson's correlation coefficient $=0.505, p=0.006$ ). No correlations were observed between the VFA and change in muscle mass (Pearson's correlation coefficient $=0.041, p=0.833$ ), the reduction in body weight and the change in SFA (Pearson's correlation coefficient $=0.170, p=0.377$ ), or the reduction in body weight and change in VFA (Pearson's correlation coefficient $=-0.203, p=0.292$ ). We identified the ipragliflozin responders as subjects with body weight reduction (1.6 kg, median value). Ipragliflozin response group tended to have higher baseline CAP and muscle mass; however, there was no clinical factor associated with ipragliflozin response in multivariable-adjusted logistic regression analyses. 
A

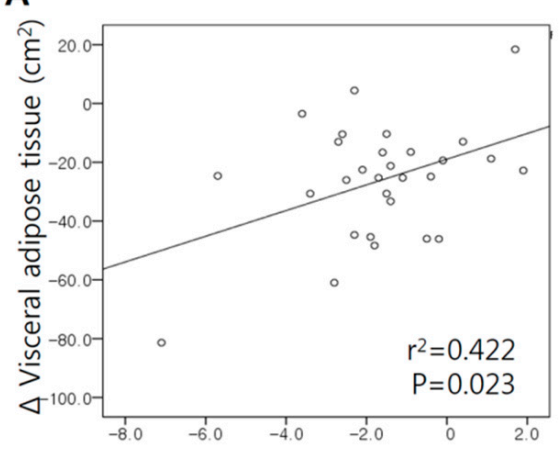

C $\Delta$ Body weight $(\mathrm{kg})$

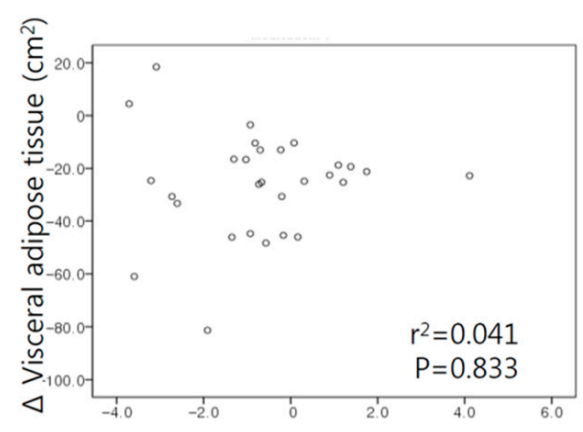

$\Delta$ Muscle mass $(\mathrm{kg})$
B

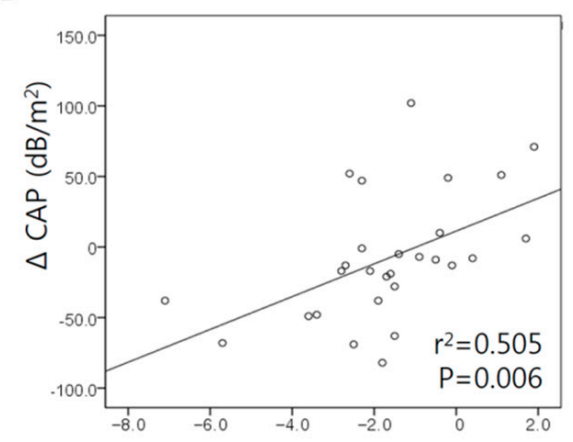

D

$\Delta$ Body weight $(\mathrm{kg})$

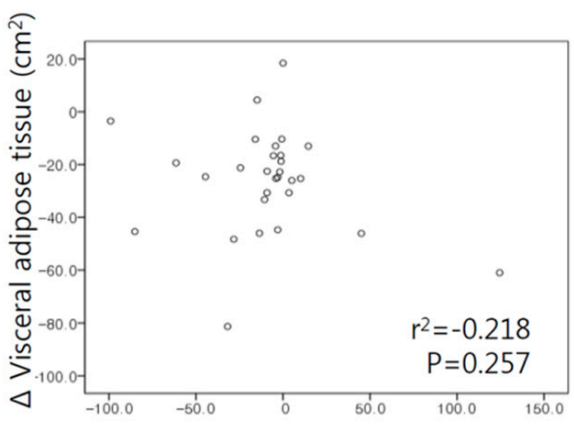

$\Delta$ Subcutaneous adipose tissue $\left(\mathrm{cm}^{2}\right)$

Figure 3. Correlation between changes in body weight, adipose tissue, liver fat and muscle mass. (A) Correlation between changes in visceral adipose tissue and body weight. (B) Correlation between changes in controlled attenuation parameter and body weight. (C) Correlation between changes in visceral adipose tissue and muscle mass. (D) Correlation between changes in visceral adipose tissue and subcutaneous adipose tissue.

\subsection{Safety $\approx$}

During the study period, 24 (61.4\%) adverse events occurred. Eight patients (18.2\%) exhibited symptoms of hypoglycemia, 11 patients $(25.0 \%)$ reported renal and urinary disorders (e.g., polyuria and pollakiuria without progression to chronic kidney disease), and 3 patients (6.8\%) had cystitis. No neoplasm development or extremity amputation occurred during the study period.

\section{Discussion}

In this open-label, randomized, controlled study, ipragliflozin as an add-on to metformin and pioglitazone in euglycemic patients with T2D and NAFLD was associated with decreased liver fat content and visceral fat depots at week 24 . Although combination metformin and pioglitazone therapy is recommended for patients with NAFLD, some patients continue to experience NAFLD progression. Ipragliflozin add-on reduced body weight, waist circumference, and visceral fat, and also improved the fatty liver index, NAFLD liver fat score, and CAP. Moreover, the reduction in body weight correlated with changes in both abdominal visceral fat and CAP. The changes in abdominal visceral fat were not associated with changes in muscle mass.

The current study has several strengths. First, although several studies have proposed that SGLT2 inhibitors lower visceral fat and provide cardiovascular protection in patients with type 2 diabetes $[13,14,25]$, this study is, as far as we know, the first to investigate the influence of SGLT2 inhibitors on whole-body fat depots and NAFLD in patients with T2D on metformin and pioglitazone maintenance therapy. The safety and efficacy of SGLT2 inhibitors as an add-on therapy to metformin and pioglitazone have been established [26-28]. These prior studies, however, have focused on the 
efficacy of SGLT2 inhibitors on reducing blood glucose and body weight. Moreover, these prior studies were conducted in patients with T2D with uncontrolled hyperglycemia (mean $\mathrm{HbA1c}$ : $7.9 \%$ to $8.1 \%$ ) despite being on metformin and pioglitazone treatment; the beneficial effects of add-on therapy may be due to glycemic reductions. In our study, patients with T2D had well-controlled $\mathrm{HbA1c}$ levels on metformin and pioglitazone (mean baseline $\mathrm{HbA} 1 \mathrm{c}$ of $6.6 \% \pm 0.6 \%$ ), which allowed us to study the impact of SGLT2 inhibitors on NAFLD rather than glycemic control. Thus, in our study, HbA1c decreased by only $0.1 \% \pm 0.2 \%$, but also showed a consistent benefit on abdominal visceral fat and other factors associated with NAFLD.

A second strength of our study is that we assessed various modalities to evaluate changes in body fat depots and the severity of NAFLD. We used both whole-body DXA scans and abdominal fat CT scans to assess fat and muscle depots and transient liver elastography and various equations to assess the severity of NAFLD. Although prior studies in animal models have reported a positive impact of SGLT2 inhibitors on body fat distribution and NAFLD severity [29,30], human studies have reported inconsistent findings. In a 102-week study, dapagliflozin add-on to metformin was associated with a $1.5 \%$ reduction in total fat mass as measured by DXA, but was not associated with changes in hepatic lipid contents as measured by magnetic resonance (MR) [31]. Another study, which compared dapagliflozin versus placebo add-on to metformin, failed to find significant changes in MR-measured VFA and SFA [32]. In these studies, however, MR was applied only in a subgroup of patients. Moreover, in the dapagliflozin versus placebo study, there was a significant difference in baseline values between the two groups.

A third strength of our study is that it showed associations between changes in adipose tissue, body weight, NAFLD indexes, and muscle mass. Although the underlying association between weight reduction and liver function improvement in response to treatment with SGLT2 inhibitors has been demonstrated in animal models $[29,33]$, associations between NAFLD severity, weight reduction, and fat distribution have not been fully elucidated in humans. In a Japanese study on the effect of SGLT2 inhibitors on liver function, a reduction in ALT was observed only in the high ALT subgroup ( $>30 \mathrm{U} / \mathrm{L}$ ), and this reduction was associated with the baseline ALT level [34]. Despite the presence of normal hepatic function at study enrollment, we demonstrated that ipragliflozin add-on consistently improved NAFLD parameters. Furthermore, improvements in CAP were associated with reductions in body weight. This finding is consistent with a two-pooled phase 3 study in which canagliflozin-related improvements in liver enzymes were associated with reductions in both body weight and $\mathrm{HbA1c}$ levels [35]. In that study, changes in liver enzymes were more strongly associated with reductions in $\mathrm{HbA1c}$ than with reductions in weight. In contrast, in our study there was no statistically significant difference in $\mathrm{HbA1c}$ levels between our two groups, which suggests that ipragliflozin additively reduces visceral fat and ameliorates NAFLD in euglycemic patients with type 2 diabetes.

Our study also has a few limitations. First, although we enrolled ultrasound-confirmed patients with NAFLD, diagnosis was not histologically confirmed. Second, although we encouraged all patients to exercise regularly and avoid overeating, we did not track food or calorie intake across the study, and thus cannot rule out the effect of these factors between the two study groups on the outcomes.

In conclusion, treatment with ipragliflozin significantly ameliorates liver steatosis and excessive fat over 24 weeks in patients with T2D and NAFLD controlled with metformin and pioglitazone. Most weight reduction was due to visceral adipose tissue loss, with significant reductions in liver fat contents. These findings provide further support for the clinical utility of ipragliflozin as an add-on therapy in patients with T2D and NAFLD.

Supplementary Materials: The following are available online at http://www.mdpi.com/2077-0383/9/1/259/s1. Figure S1. Study flow diagram; Table S1. Non-invasive calculation methods for assessing liver steatosis.

Author Contributions: Conception and design, E.H., Y.-h.L., and B.-S.C.; development of methodology, E.H., Y.-h.L., and B.-S.C.; analysis and interpretation of data, E.H., Y.-h.L., and B.-S.C.; writing, review, and/or revision of the manuscript, E.H., Y.-h.L., B.-W.L., E.S.K., and B.-S.C.; administrative, technical, or material support, E.H., Y.-h.L., and B.-S.C.; study supervision, B.-S.C. All authors have read and agreed to the published version of the manuscript. 
Funding: Sponsorship for this study and article processing charges were funded by Astellas Pharma Korea, Inc. Conflicts of Interest: The authors declare no conflicts of interest.

$\begin{array}{ll}\text { Abbreviations } & \\ \text { DXA } & \begin{array}{l}\text { dual-energy x-ray absorptiometry } \\ \text { estimated glomerular filtration rate } \\ \text { fGFR }\end{array} \\ \text { FPG } & \begin{array}{l}\text { glycated hemoglobin } \\ \text { high-density lipoprotein cholesterol }\end{array} \\ \text { HbA1c } & \text { homeostasis model assessment of insulin resistance } \\ \text { HDL-C } & \text { low-density lipoprotein cholesterol } \\ \text { HOMA-IR } & \text { non-alcoholic fatty liver disease; } \\ \text { LDL-C } & \text { sodium/glucose cotransporter 2; } \\ \text { NAFLD } & \text { subcutaneous fat area } \\ \text { SGLT2 } & \text { thiazolidinedione } \\ \text { SFA } & \text { visceral fat area } \\ \text { TZD } & \end{array}$

\section{References}

1. Tilg, H.; Moschen, A.R.; Roden, M. NAFLD and diabetes mellitus. Nat. Rev. Gastroenterol. Hepatol. 2017, 14, 32-42. [CrossRef] [PubMed]

2. Yoon, H.J.; Lee, Y.-H.; Cha, B.-S. Causal relationship of non-alcoholic fatty liver disease with obesity and insulin resistance. J. Korean Diabetes 2014, 15, 76-81. [CrossRef]

3. Han, E.; Lee, Y.H. Non-Alcoholic Fatty Liver Disease: The Emerging Burden in Cardiometabolic and Renal Diseases. Diabetes Metab. J. 2017, 41, 430-437. [CrossRef] [PubMed]

4. European Association for the Study of the Liver and European Association for the Study of Diabetes. EASL-EASD-EASO Clinical Practice Guidelines for the management of non-alcoholic fatty liver disease. Obes. Facts 2016, 9, 65-90. [CrossRef]

5. Lee, Y.H.; Kim, J.H.; Kim, S.R.; Jin, H.Y.; Rhee, E.J.; Cho, Y.M.; Lee, B.W. Lobeglitazone, a Novel Thiazolidinedione, Improves Non-Alcoholic Fatty Liver Disease in Type 2 Diabetes: Its Efficacy and Predictive Factors Related to Responsiveness. J. Korean Med. Sci. 2017, 32, 60-69. [CrossRef]

6. Han, E.; Jang, S.Y.; Kim, G.; Lee, Y.H.; Choe, E.Y.; Nam, C.M.; Kang, E.S. Rosiglitazone Use and the Risk of Bladder Cancer in Patients With Type 2 Diabetes. Medicine 2016, 95, e2786. [CrossRef]

7. DeFronzo, R.A.; Chilton, R.; Norton, L.; Clarke, G.; Ryder, R.E.; Abdul-Ghani, M. Revitalization of pioglitazone: The optimum agent to be combined with a sodium-glucose co-transporter-2 inhibitor. Diabetes Obes. Metab. 2016, 18, 454-462. [CrossRef]

8. Belfort, R.; Harrison, S.A.; Brown, K.; Darland, C.; Finch, J.; Hardies, J.; Balas, B.; Gastaldelli, A.; Tio, F.; Pulcini, J.; et al. A placebo-controlled trial of pioglitazone in subjects with nonalcoholic steatohepatitis. N. Engl. J. Med. 2006, 355, 2297-2307. [CrossRef]

9. Basu, A.; Jensen, M.D.; McCann, F.; Mukhopadhyay, D.; Joyner, M.J.; Rizza, R.A. Effects of pioglitazone versus glipizide on body fat distribution, body water content, and hemodynamics in type 2 diabetes. Diabetes Care 2006, 29, 510-514. [CrossRef]

10. Ratziu, V.; Giral, P.; Jacqueminet, S.; Charlotte, F.; Hartemann-Heurtier, A.; Serfaty, L.; Podevin, P.; Lacorte, J.M.; Bernhardt, C.; Bruckert, E.; et al. Rosiglitazone for nonalcoholic steatohepatitis: One-year results of the randomized placebo-controlled Fatty Liver Improvement with Rosiglitazone Therapy (FLIRT) Trial. Gastroenterology 2008, 135, 100-110. [CrossRef]

11. Sanyal, A.J.; Chalasani, N.; Kowdley, K.V.; McCullough, A.; Diehl, A.M.; Bass, N.M.; Neuschwander-Tetri, B.A.; Lavine, J.E.; Tonascia, J.; Unalp, A.; et al. Pioglitazone, Vitamin E or placebo for nonalcoholic steatohepatitis. N. Engl. J. Med. 2010, 362, 1675-1685. [CrossRef]

12. Jung, C.H.; Jang, J.E.; Park, J.Y. A Novel Therapeutic Agent for Type 2 Diabetes Mellitus: SGLT2 Inhibitor. Diabetes Metab. J. 2014, 38, 261-273. [CrossRef] 
13. Zinman, B.; Wanner, C.; Lachin, J.M.; Fitchett, D.; Bluhmki, E.; Hantel, S.; Mattheus, M.; Devins, T.; Johansen, O.E.; Woerle, H.J.; et al. Empagliflozin, Cardiovascular Outcomes, and Mortality in Type 2 Diabetes. N. Engl. J. Med. 2015, 373, 2117-2128. [CrossRef]

14. Neal, B.; Perkovic, V.; Mahaffey, K.W.; de Zeeuw, D.; Fulcher, G.; Erondu, N.; Shaw, W.; Law, G.; Desai, M.; Matthews, D.R. Canagliflozin and Cardiovascular and Renal Events in Type 2 Diabetes. N. Engl. J. Med. 2017, 377, 644-657. [CrossRef]

15. Han, E.; Shin, E.; Kim, G.; Lee, J.-Y.; Lee, Y.-H.; Lee, B.-W.; Kang, E.S.; Cha, B.S. Combining SGLT2 inhibition with a thiazolidinedione additively attenuate the very early phase of diabetic nephropathy progression in type 2 diabetes mellitus. Front. Endocrinol. 2018, 9, 412. [CrossRef]

16. Fioretto, P.; Zambon, A.; Rossato, M.; Busetto, L.; Vettor, R. SGLT2 Inhibitors and the Diabetic Kidney. Diabetes Care 2016, 39 (Suppl. S2), S165-S171. [CrossRef]

17. Han, E.; Kim, A.; Lee, S.J.; Kim, J.Y.; Kim, J.H.; Lee, W.J.; Lee, B.W. Characteristics of Dapagliflozin Responders: A Longitudinal, Prospective, Nationwide Dapagliflozin Surveillance Study in Korea. Diabetes Ther. 2018, 9, 1689-1701. [CrossRef]

18. Komiya, C.; Tsuchiya, K.; Shiba, K.; Miyachi, Y.; Furuke, S.; Shimazu, N.; Yamaguchi, S.; Kanno, K.; Ogawa, Y. Ipragliflozin Improves Hepatic Steatosis in Obese Mice and Liver Dysfunction in Type 2 Diabetic Patients Irrespective of Body Weight Reduction. PLoS ONE 2016, 11, e0151511. [CrossRef]

19. Min, K.W.; Ku, B.J.; Lee, J.H.; Kim, M.S.; Ahn, K.J.; Lee, M.K.; Kokubo, S.; Yoshida, S.; Cho, H.J.; Cha, B.S. Addition of Ipragliflozin to Metformin Treatment in Korean Patients with Type 2 Diabetes Mellitus: Subgroup Analysis of a Phase 3 Trial. Diabetes Metab. J. 2017, 41, 135-145. [CrossRef]

20. Chon, Y.E.; Jung, K.S.; Kim, S.U.; Park, J.Y.; Park, Y.N.; Kim, D.Y.; Ahn, S.H.; Chon, C.Y.; Lee, H.W.; Park, Y.; et al. Controlled attenuation parameter (CAP) for detection of hepatic steatosis in patients with chronic liver diseases: A prospective study of a native Korean population. Liver Int. 2014, 34, 102-109. [CrossRef]

21. Bedogni, G.; Bellentani, S.; Miglioli, L.; Masutti, F.; Passalacqua, M.; Castiglione, A.; Tiribelli, C. The Fatty Liver Index: A simple and accurate predictor of hepatic steatosis in the general population. BMC Gastroenterol. 2006, 6, 33. [CrossRef]

22. Kotronen, A.; Peltonen, M.; Hakkarainen, A.; Sevastianova, K.; Bergholm, R.; Johansson, L.M.; Lundbom, N.; Rissanen, A.; Ridderstråle, M.; Groop, L.; et al. Prediction of non-alcoholic fatty liver disease and liver fat using metabolic and genetic factors. Gastroenterology 2009, 137, 865-872. [CrossRef]

23. Lee, J.W.; Lee, H.R.; Shim, J.Y.; Im, J.A.; Kim, S.H.; Choi, H.; Lee, D.C. Viscerally obese women with normal body weight have greater brachial-ankle pulse wave velocity than nonviscerally obese women with excessive body weight. Clin. Endocrinol. 2007, 66, 572-578. [CrossRef]

24. Lee, J.Y.; Lee, H.S.; Lee, D.C.; Chu, S.H.; Jeon, J.Y.; Kim, N.K.; Lee, J.W. Visceral fat accumulation is associated with colorectal cancer in postmenopausal women. PLoS ONE 2014, 9, e110587. [CrossRef]

25. Radholm, K.; Figtree, G.; Perkovic, V.; Solomon, S.D.; Mahaffey, K.W.; de Zeeuw, D.; Fulcher, G.; Barrett, T.D.; Shaw, W.; Desai, M.; et al. Canagliflozin and Heart Failure in Type 2 Diabetes Mellitus: Results From the CANVAS Program. Circulation 2018, 138, 458-468. [CrossRef]

26. Forst, T.; Guthrie, R.; Goldenberg, R.; Yee, J.; Vijapurkar, U.; Meininger, G.; Stein, P. Efficacy and safety of canagliflozin over 52 weeks in patients with type 2 diabetes on background metformin and pioglitazone. Diabetes Obes. Metab. 2014, 16, 467-477. [CrossRef]

27. Kovacs, C.S.; Seshiah, V.; Swallow, R.; Jones, R.; Rattunde, H.; Woerle, H.J.; Broedl, U.C. Empagliflozin improves glycaemic and weight control as add-on therapy to pioglitazone or pioglitazone plus metformin in patients with type 2 diabetes: A 24-week, randomized, placebo-controlled trial. Diabetes Obes. Metab. 2014, 16, 147-158. [CrossRef]

28. Kovacs, C.S.; Seshiah, V.; Merker, L.; Christiansen, A.V.; Roux, F.; Salsali, A.; Kim, G.; Stella, P.; Woerle, H.J. Empagliflozin as Add-on Therapy to Pioglitazone with or without Metformin in Patients with Type 2 Diabetes Mellitus. Clin. Ther. 2015, 37, 1773-1788. [CrossRef]

29. Honda, Y.; Imajo, K.; Kato, T.; Kessoku, T.; Ogawa, Y.; Tomeno, W.; Kato, S.; Mawatari, H.; Fujita, K.; Yoneda, M.; et al. The Selective SGLT2 Inhibitor Ipragliflozin Has a Therapeutic Effect on Nonalcoholic Steatohepatitis in Mice. PLoS ONE 2016, 11, e0146337. [CrossRef]

30. Watanabe, Y.; Nakayama, K.; Taniuchi, N.; Horai, Y.; Kuriyama, C.; Ueta, K.; Arakawa, K.; Senbonmatsu, T.; Shiotani, M. Beneficial effects of canagliflozin in combination with pioglitazone on insulin sensitivity in rodent models of obese type 2 diabetes. PLOS ONE 2015, 10, e0116851. [CrossRef] 
31. Bolinder, J.; Ljunggren, O.; Johansson, L.; Wilding, J.; Langkilde, A.M.; Sjostrom, C.D.; Sugg, J.; Parikh, S. Dapagliflozin maintains glycaemic control while reducing weight and body fat mass over 2 years in patients with type 2 diabetes mellitus inadequately controlled on metformin. Diabetes Obes. Metab. 2014, 16, 159-169. [CrossRef] [PubMed]

32. Bolinder, J.; Ljunggren, O.; Kullberg, J.; Johansson, L.; Wilding, J.; Langkilde, A.M.; Sugg, J.; Parikh, S. Effects of dapagliflozin on body weight, total fat mass, and regional adipose tissue distribution in patients with type 2 diabetes mellitus with inadequate glycemic control on metformin. J. Clin. Endocrinol. Metab. 2012, 97, 1020-1031. [CrossRef] [PubMed]

33. Tahara, A.; Kurosaki, E.; Yokono, M.; Yamajuku, D.; Kihara, R.; Hayashizaki, Y.; Takasu, T.; Imamura, M.; Li, Q.; Tomiyama, H.; et al. Effects of sodium-glucose cotransporter 2 selective inhibitor ipragliflozin on hyperglycaemia, oxidative stress, inflammation and liver injury in streptozotocin-induced type 1 diabetic rats. J. Pharm. Pharmacol. 2014, 66, 975-987. [CrossRef] [PubMed]

34. Seko, Y.; Sumida, Y.; Sasaki, K.; Itoh, Y.; Iijima, H.; Hashimoto, T.; Ishii, S.; Inagaki, N. Effects of canagliflozin, an SGLT2 inhibitor, on hepatic function in Japanese patients with type 2 diabetes mellitus: Pooled and subgroup analyses of clinical trials. J. Gastroenterol. 2018, 53, 140-151. [CrossRef]

35. Leiter, L.A.; Forst, T.; Polidori, D.; Balis, D.A.; Xie, J.; Sha, S. Effect of canagliflozin on liver function tests in patients with type 2 diabetes. Diabetes Metab. 2016, 42, 25-32. [CrossRef]

(C) 2020 by the authors. Licensee MDPI, Basel, Switzerland. This article is an open access article distributed under the terms and conditions of the Creative Commons Attribution (CC BY) license (http://creativecommons.org/licenses/by/4.0/). 